\title{
Detection of natural antisense non-coding RNAs transcribed from Ncam1 in mice tissues at several developmental stages
}

\author{
MITSURU CHIBA \\ Department of Biomedical Sciences, Division of Medical Life Sciences, Hirosaki University \\ Graduate School of Health Sciences, Hirosaki, Aomori 036-8564, Japan
}

Received April 29, 2015; Accepted May 21, 2015

DOI: $10.3892 /$ br.2015.482

\begin{abstract}
Natural antisense ribonucleic acids (RNAs) are transcribed from a large number of genes in various species, including humans and mice. The expression of neural cell adhesion molecule 1 (Ncaml) antisense non-coding RNAs (ncRNAs) in mice has been demonstrated by functional annotation of the mammalian genome project, but the localization of Ncaml antisense ncRNAs has not been reported in mice tissues. In the present study, the localization of Ncaml antisense ncRNAs was examined in tissues at several developmental stages by in situ hybridization. At days 14 and 17 of embryonic development, Ncaml antisense ncRNAs were found in the heart, liver, lung, kidney, thymus and nerve regions including the brain (cerebral cortex, olfactory bulb, hippocampus and cerebellum) and spinal cord. In newborn mice, Ncaml antisense ncRNAs were detected in the brain, kidney and thymus, but was not detected in other tissues. In 8-week-old mice, Ncaml antisense ncRNAs were detected in the lung, kidney, thymus, pancreas, cornea, stomach and nerve regions including the brain. These results indicate that Ncaml antisense ncRNAs are expressed in mice tissues. Notably, Ncaml messenger RNAs (mRNAs), antisense ncRNAs co-localized in the Purkinje cells of the cerebellum and the levels of antisense ncRNAs appeared to be higher than those of mRNAs, suggesting that Ncaml antisense ncRNAs may regulate the expression of $N$ caml mRNAs in the same cells.
\end{abstract}

\section{Introduction}

Natural antisense ribonucleic acids (RNAs) are transcripts that contain sequences complementary to other endogenous RNAs including messenger RNAs (mRNAs). Kiyosawa et al $(1,2)$ identified $\sim 2,500$ complementary deoxyribonucleic acid

Correspondence to: Dr Mitsuru Chiba, Department of Biomedical Sciences, Division of Medical Life Sciences, Hirosaki University Graduate School of Health Sciences, 66-1 Hon-cho, Hirosaki, Aomori 036-8564, Japan

E-mail: mchiba32@hirosaki-u.ac.jp

Key words: neural cell adhesion molecule 1, natural antisense non-coding RNAs, in situ hybridization, embryo, Purkinje cell
(cDNA) pairs originating from opposite strands of the same genomic loci by mapping these cDNAs to the draft mouse genome sequence. The functional annotation of the mammalian genome (FANTOM) cDNA dataset contained numerous non-coding RNAs (ncRNAs) (1-3). More than $70 \%$ of the antisense pairs identified by Kiyosawa et al $(1,2)$ included ncRNAs as one of the members in the pair. To date, a number of mammalian antisense ncRNAs have been found, indicating that antisense transcription may be a commonly employed mechanism to regulate gene expression in human cells (1-5). Faghihi et al (6) reviewed the functions of known antisense ncRNAs, such as transcriptional interference, genomic imprinting, $\mathrm{X}$ chromosome inactivation and alternative splicing. Certain investigators have reported that the gene expression of antisense ncRNAs was up- or downregulated under conditions of physiological change, such as the development of various types of cancer $(7,8)$. However, it is difficult to elucidate the function of antisense ncRNAs via gene expression analyses, such as microarrays.

The neural cell adhesion molecule 1 (Ncaml, also known as CD56) gene encodes a cell adhesion protein and is a member of the immunoglobulin superfamily. The encoded protein is involved in cell-to-cell and cell-matrix interactions during development and differentiation, as well as nervous system development $(9,10)$. Antisense ncRNAs from opposite strands of the Ncaml loci in mice have been registered in the NCBI database (http://www.ncbi.nlm.nih.gov/gene/100036537) and antisense viewer (http://genome.gsc.riken.jp/m/antisense). In addition, the expression of antisense ncRNAs from Ncaml loci in the brain has been proven using microarray analysis by Kiyosawa et al (2). However, the expression and localization of this ncRNAs in other tissues remains unclear.

It is necessary to examine the cells and/or tissues from which the ncRNAs are expressed in order to understand the role of Ncaml antisense ncRNAs. In the present study, the expression of Ncaml antisense ncRNAs was examined in mice tissues at different developmental stages by in situ hybridization.

\section{Materials and methods}

Sections of the embryo and tissues. C57BL/6J mice at different developmental stages (at day 14 of mouse embryo development, E14; day 17 of development, E17; and newborn 
mice; 8-week-old mice) were obtained from the RIKEN BioResource Center (Ibaraki, Japan).

For in situ hybridization, tissues from mice at different developmental stages were first fixed in situ by perfusion with $4 \%(\mathrm{w} / \mathrm{v})$ ice-cold paraformaldehyde solution in phosphate-buffered saline (PBS). The resulting tissues were excised and further fixed overnight in the paraformaldehyde solution. The fixed tissues were embedded in paraffin and $4 \mu \mathrm{m}$ sections were cut. Sections were placed on glass slides and subjected to in situ hybridization.

Complementary RNA (cRNA) probe. For Ncaml antisense ncRNAs and mRNAs, probes with a specific sequence: 5'-ATC TGGTCAAGTACAGAGCGCTCGCCTCTGAGTGGAAAC CGGAAATCAGGCTCCCATCCGGCAGTGACGACC ACGTCATGCTCAAGTCCCTGGACTGGAACGCAGAGT ATGAAGTCTATG T-3'; and a size of 118 nucleotides were designed in the mRNAs of Ncaml. As a control RNA probe, a 120 -nucleotide $\lambda$-phage sequence that had no similarity with any of the mammalian sequences registered in the DNA Data Bank of Japan (www.ddbj.nig.ac.jp/index-j.html) was used in all the in situ hybridization experiments to verify that the hybridization system did not emit any non-specific hybridization signals. Digoxigenin (DIG)-labeled cRNA probes were provided by Tsukuba GeneTech Laboratories (Ibaraki, Japan).

In situ hybridization. Sections of embryos and tissues on glass slides were deparrafinized with xylene and ethanol, washed in PBS and incubated in PBS containing $1.0 \mu \mathrm{g} / \mathrm{ml}$ proteinase $\mathrm{K}$, at $37^{\circ} \mathrm{C}$ for $15 \mathrm{~min}$. The slides were washed in PBS and treated with $0.1 \mathrm{M}$ triethanolamine in $0.25 \%$ acetic anhydride for $15 \mathrm{~min}$. Slides were subsequently washed with $0.1 \mathrm{M}$ triethanolamine and 4X standard saline citrate (SSC), and incubated in prehybridization solution (50\% formamide, 2X SSC) at $42^{\circ} \mathrm{C}$ for $30 \mathrm{~min}$. Hybridization was performed in a solution containing $50 \%$ formamide, $2 X \mathrm{SSC}, 1.0 \mathrm{mg} / \mathrm{ml}$ transfer RNA (tRNA), $1.0 \mathrm{mg} / \mathrm{ml}$ salmon DNA, $1.0 \mathrm{mg} / \mathrm{ml}$ bovine serum albumin, $1.0 \%$ sodium dodecyl sulfate and $3.0 \mu \mathrm{g} / \mathrm{ml}$ DIG-labeled sense or antisense cRNA probes, at $42^{\circ} \mathrm{C}$ for $16 \mathrm{~h}$.

The resulting slides were successively washed with prehybridization solution, $0.2 \mathrm{X} \mathrm{SSC}$ and $0.1 \mathrm{X} \mathrm{SSC}$, at $42^{\circ} \mathrm{C}$, followed by treatment with NT buffer $[150 \mathrm{mmol} / 1 \mathrm{NaCl}$ and $100 \mathrm{mmol} / \mathrm{l}$ Tris-HCl (pH 7.5)] containing $10 \%$ fetal bovine serum, $1 \%$ blocking reagent (Roche Diagnostics, Basel, Switzerland) and $1 \mathrm{mg} / \mathrm{ml}$ tRNA, for $30 \mathrm{~min}$. Subsequently, hybridization signals were visualized using the alkaline phosphatase-labeled anti-DIG antibody/nitro blue tetrazolium chloride 5-bromo-4-chloro-3-indolyl-phosphate system (Roche Diagnostics).

\section{Results and Discussion}

Recently, antisense ncRNAs from a number of genes have been identified in mice and humans using bioinformatic and microarray analyses. Antisense ncRNAs have been inferred to be involved in the control of trait expression in mammals, including humans, mice and livestock. Study of the sites of antisense ncRNA expression in tissues would provide important information regarding the functions of antisense ncRNAs in mice. Therefore, mice were used in the present study to investigate the expression sites of previously described antisense ncRNAs from Ncaml.

Kiyosawa et al (2) examined sense/antisense expression using a custom microarray with different cDNA priming methods. Ncaml antisense ncRNAs in the brain were more strongly expressed than those mRNAs in the microarray analysis using oligo(dT) or random nonamer primers (Fig. 1). Microarray analysis using oligo(dT) primers is assumed to detect RNAs (transcripts) with poly(A) tails, whereas microarray analysis using random nonamer primers is assumed to detect RNAs with and without poly(A) tails. This information suggests that Ncaml antisense ncRNAs may have poly(A) tails.

To date, the open reading frame, or protein-coding region, of Ncaml antisense RNAs has not been confirmed in several databases, including FANTOM. Therefore, Ncaml antisense RNAs are ncRNAs. To examine the expression and localization of Ncaml antisense ncRNAs at different developmental stages, in situ hybridization was performed. In situ hybridization is known to detect RNAs with and without poly(A) tails. Therefore, it is generally considered that the signal intensity patterns of in situ hybridization are the same as those of microarray analysis using random nonamer primers.

In E14 embryos, Ncaml antisense ncRNAs were uniformly detected in the heart, liver and nervous system regions, including the brain, and were detected at high levels in certain regions of the lung, kidney and thymus (Fig. 2). In E17 embryos, Ncaml antisense ncRNAs were detected at high levels in specific cells of the brain, whereas they were uniformly detected in other tissues (heart, liver, lung, kidney and thymus). In newborn mice, Ncaml antisense ncRNAs were weakly detected in the brain, kidney and thymus. The transcription pattern of NB mice was similar to that of E17 embryos, except that the signals in organs other than the brain were weaker than those of the E17 embryos (Figs. 2 and 3). In the 8-week-old mice, Ncaml antisense ncRNAs were detected at high levels in limited regions of the brain, stomach, cornea and all the regions of the pancreas. Contrastingly, they were detected at low levels in the remaining organs that were examined, excluding the liver. With regards to the liver, negligible amounts of Ncaml antisense ncRNA expression was detected (Fig. 3). These results indicate that Ncaml antisense ncRNAs are expressed in mice tissues.

Of note, Ncaml mRNAs and antisense ncRNAs co-localized in Purkinje cells of the cerebellum and the levels of antisense ncRNAs appeared to be higher than those of mRNAs (Fig. 4). Based on the patterns of the in situ hybridization signals for Ncaml antisense ncRNAs detected in tissues, some potential functions were identified for Ncaml antisense ncRNAs. In the analysis of the adjacent sections, Ncaml mRNAs were detected in some of the brain cells found to produce Ncaml antisense ncRNAs. This indicates that Ncaml antisense ncRNAs may control the function of Ncaml mRNAs, as previously proposed in reviews on other antisense ncRNAs $(6,11)$. Currently, the exact function of Ncaml antisense ncRNAs in each tissue is unknown. However, the expression patterns of Ncaml antisense ncRNAs may provide information for understanding their function. In future studies, the interactions between Ncaml mRNAs and antisense ncRNAs should be examined. 

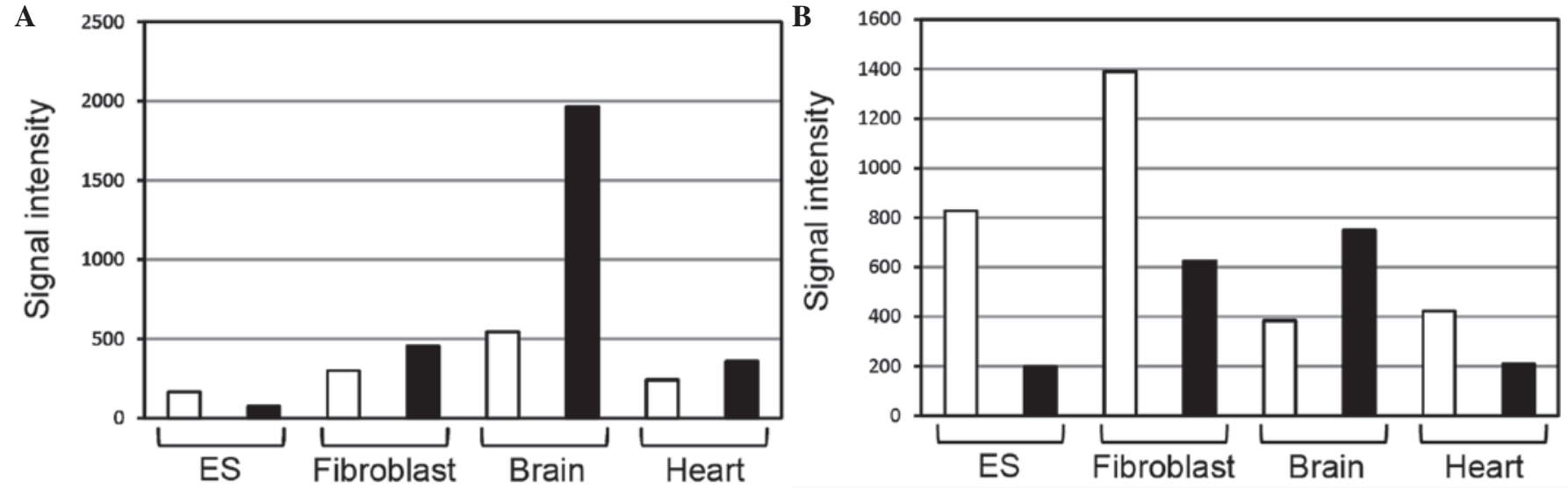

Figure 1. Expression of neural cell adhesion molecule 1 (Ncaml) messenger RNAs (mRNAs) and antisense non-coding RNAs (ncRNAs), according to microarray analysis. (A) Microarray signal intensity obtained with samples labeled by oligo(dT) priming. (B) Microarray signal intensity obtained with samples labeled by random nonamer priming. White bars represent the signal intensity of Ncaml mRNAs, whereas black bars represent the signal intensity of Ncam 1 antisense ncRNAs.
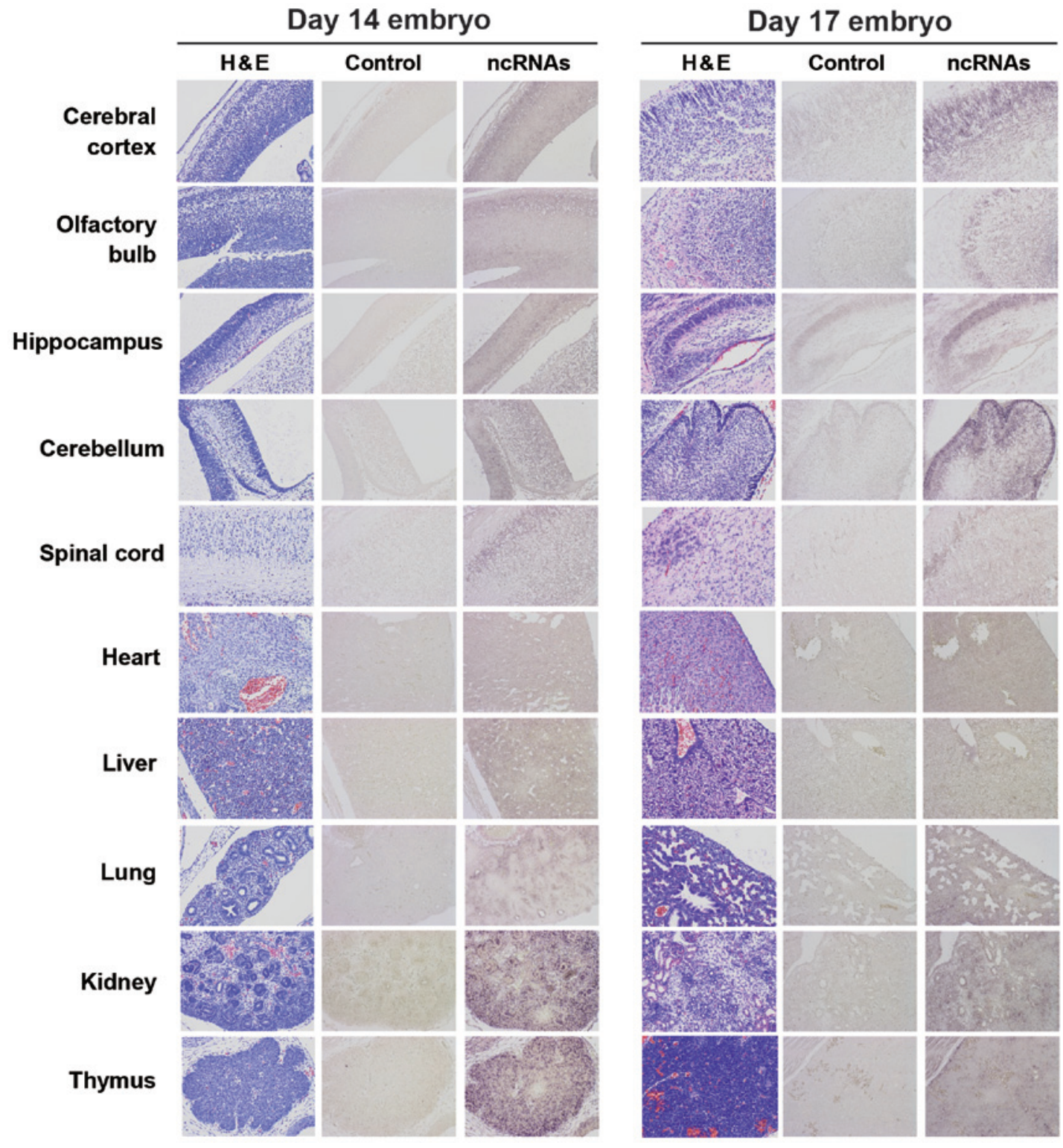

Figure 2. Detection of neural cell adhesion molecule 1 (Ncaml) antisense non-coding RNAs (ncRNAs) in mice tissues at embryonic development day 14 (E14) and 17 (E17). Ncaml antisense ncRNAs were detected by in situ hybridization with a digoxigenin (DIG)-labeled sense complementary-RNA probe. As a negative control, DIG-labeled control probes were used for in situ hybridization. Hematoxylin and eosin (H\&E) staining was performed to visualize the fine structures of the tissues. 


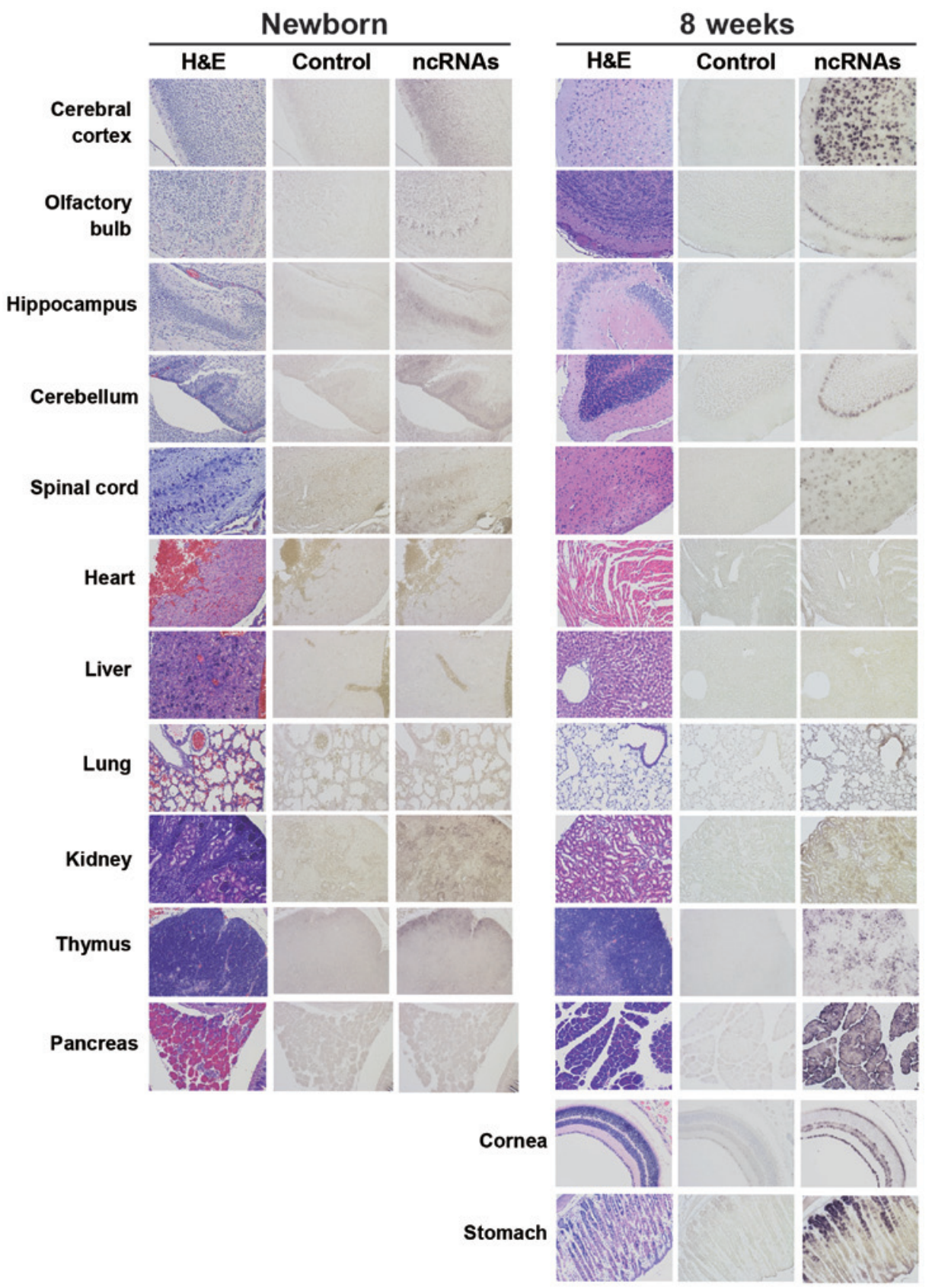

Figure 3. Detection of neural cell adhesion molecule 1 (Ncam1) antisense non-coding RNAs (ncRNAs) in newborn and 8-week-old mice. Ncam1 antisense ncRNAs were detected by in situ hybridization with a digoxigenin (DIG)-labeled sense complementary-RNA probe. As a negative control, DIG-labeled control probes were used for in situ hybridization. Hematoxylin and eosin (H\&E) staining was performed to visualize the fine structures of tissues.
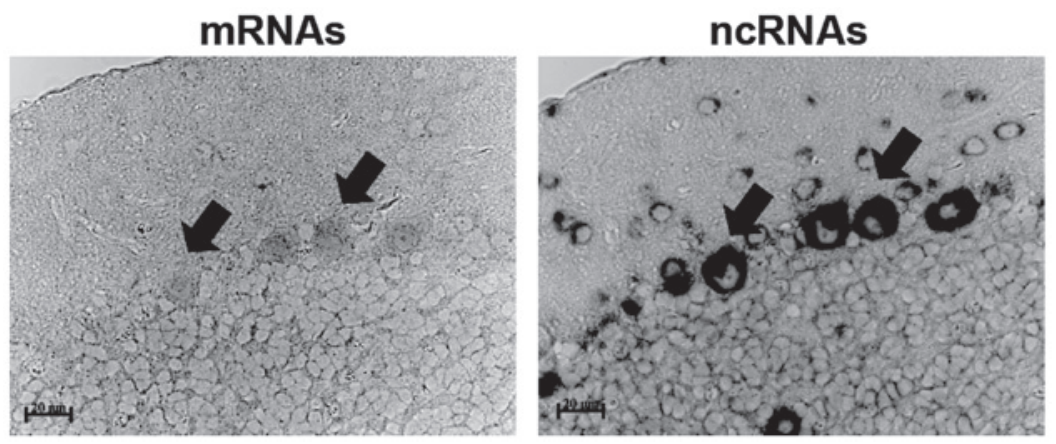

Figure 4. Co-localization of neural cell adhesion molecule 1 (Ncam1) messenger RNAs (mRNAs) and antisense non-coding RNAs (ncRNAs) in Purkinje cells of 8 -week-old mice. Murine cerebellum tissue embedded in paraffin was serially sectioned at $1.5-\mu \mathrm{m}$, which allowed one Purkinje cell to be split into $\geq 2$ consecutive sections. In situ hybridization was performed using a digoxigenin-labeled sense complementary-RNA probe. Ncaml mRNAs and antisense ncRNAs existed in the same Purkinje cells, as indicated by black arrows. Scale bar represents $20 \mu \mathrm{m}$. 


\section{Acknowledgements}

The author would like to thank Dr Hiroshi Yasue of Tsukuba GeneTech Laboratories (Ibaraki, Japan) for the provision of DIG-labeled cRNA probes and Ms. Noriko Hiraiwa of RIKEN BioResource Center for offering the samples. The present study was supported in part by the Grants in Aid from the Ministry of Education, Culture, Sports, Science and Technology of Japan and the Hirosaki University Grant for Exploratory Research by Young Scientists.

\section{References}

1. Kiyosawa H, Yamanaka I, Osato N, Kondo S and Hayashizaki Y RIKEN GER Group; GSL Members: Antisense transcripts with FANTOM2 clone set and their implications for gene regulation. Genome Res 13 (6B): 1324-1334, 2003.

2. Kiyosawa H, Mise N, Iwase S, Hayashizaki Y and Abe K: Disclosing hidden transcripts: Mouse natural sense-antisense transcripts tend to be poly(A) negative and nuclear localized. Genome Res 15: 463-474, 2005.

3. Okazaki Y, Furuno M, Kasukawa T, Adachi J, Bono H, Kondo S, Nikaido I, Osato N, Saito R, Suzuki H, et al; FANTOM Consortium; RIKEN Genome Exploration Research Group Phase I \& II Team: Analysis of the mouse transcriptome based on functional annotation of 60,770 full-length cDNAs. Nature 420: 563-573, 2002.
4. Katayama S, Tomaru Y, Kasukawa T, Waki K, Nakanishi M, Nakamura M, Nishida H, Yap CC, Suzuki M, Kawai J, et al; FANTOM Consortium: Antisense transcription in the mammalian transcriptome. Science 309: 1564-1566, 2005.

5. Lehner B, Williams G, Campbell RD and Sanderson CM: Antisense transcripts in the human genome. Trends Genet 18: 63-65, 2002.

6. Faghihi MA and Wahlestedt C: Regulatory roles of natural antisense transcripts. Nat Rev Mol Cell Biol 10: 637-643, 2009.

7. Kohno K, Chiba M, Murata S, Pak S, Nagai K, Yamamoto M, Yanagisawa K, Kobayashi A, Yasue H and Ohkohchi N: Identification of natural antisense transcripts involved in human colorectal cancer development. Int J Oncol 37: 1425-1432, 2010.

8. Grigoriadis A, Oliver GR, Tanney A, Kendrick H, Smalley MJ Jat P and Neville AM: Identification of differentially expressed sense and antisense transcript pairs in breast epithelial tissues. BMC Genomics 10: 324, 2009.

9. Cremer H, Lange R, Christoph A, Plomann M, Vopper G, Roes J, Brown R, Baldwin S, Kraemer P, Scheff S, et al: Inactivation of the N-CAM gene in mice results in size reduction of the olfactory bulb and deficits in spatial learning. Nature 367: 455-459, 1994.

10. Dityatev A, Dityateva G, Sytnyk V, Delling M, Toni N, Nikonenko I, Muller D and Schachner M: Polysialylated neural cell adhesion molecule promotes remodeling and formation of hippocampal synapses. J Neurosci 24: 9372-9382, 2004.

11. Lavorgna G, Dahary D, Lehner B, Sorek R, Sanderson CM and Casari G: In search of antisense. Trends Biochem Sci 29: 88-94, 2004. 